\title{
Cultural Development of Mathematical Ideas: Papua New Guinea Studies
}

\author{
Geoffrey Saxe, with Indigo Esmonde Cambridge University Press, 2012, (hardback), ISBN: 9780521761666. \\ Reviewed by Kay Owens, Charles Sturt University, Locked Bag 49, Dubbo 2830, Australia. Email: kowens@csu.edu.au \\ Visiting Scholar, Glen Lean Ethnomathematics Centre, University of Goroka, Papua New Guinea
}

\begin{abstract}
This review highlights the strengths of Geoffrey Saxe's latest book on cultural development of mathematical ideas. His research was undertaken in a remote area of Papua New Guinea. This book is important for it brings together Saxe's critical thinking on genetic development within the field of cognitive psychology as it relates to mathematics. This is an important contribution to ethnomathematics.
\end{abstract} Keywords: ethnomathematics, cognitive development, mathematics learning, ecocultural development, Papua
New Guinea

Saxe's previous book on Culture and Cognitive Development (Saxe, 1991) provided fascinating discussion of how sociocultural experiences impact on ways of thinking. His study in Brazil considered the form used by different cohorts of children to recognise and compare different monetary values, and how they added or subtracted different amounts. He compared responses of young streetsellers in different cohorts (age and rural/nonrural; with and without schooling) and non-streetsellers who did go to school. Interestingly, he showed that sellers, especially younger ones, used the money (paper notes) rather than the school-type numeral representation as the form for arithmetic functions to varying degrees of effectiveness. Furthermore, he suggests that what is traditionally considered as learning transfer is well understood as an interplay between learning across cultural practices.

In his recent book, Cultural Development of Mathematical Ideas, Saxe develops his argument about the developmental origins of cognition. This genetic approach to understanding cognition is substantiated by a systematic and insightful series of analyses over time and within time periods. He develops a clear diagram to summarise these findings and to bring the complexities of his genetic theory together in an interwoven manner. Saxe first challenges the idea that signs and systems amplify and extend thought. He suggests this idea is inadequate to explain change because of the variance within circumstances and systems. Culture and cognition cannot be kept separate from the sociohistorical cultural and cognitive developmental processes. Both culture and cognition participate in constituting the other as processes that respond to vari- ations in culture over time. Saxe's book clearly justifies his theory. Furthermore, he shows that the boundaries of culture are osmotic, resulting in change and variance in representations, practices, and artefacts, and yet there is also diversity within the culture.

Saxe has written clearly, engaging the reader in the first chapter with his personal journey into a little known realm of the world, the Oksapmin of the highland reaches of the west Sepik area of Papua New Guinea (PNG). He follows this with his theoretical journey, referring to various theories that approach psychology from an anthropological or cognitive constructivist perspective to present his genetic model. As a process, cognition is not merely internal as representation is part of the cognitive process; and it has cultural forms and cognitive functions for both individual and group activity. Form-function relations as processes in motion are constituted and shifted in the processes of microgenesis, sociogenesis and ontogenesis. His model incorporates microgenetic development at the personal level and how this relates to the sociogenetic level as individuals in the society influence each other. Over time at a personal level, there is an ontogenetic development that leads to changes in the individual and hence at the social level. This criss-cross influence is illustrated diagrammatically on p. 33 of his book. At the microgenetic level, change is not a mere transmission from individual to individual as in one person suggesting a new use for a particular idea, but change for the individual is the result of societal development in the construction and use of ideas and their representations and modifications. This is the sociogenetic level. These social constructions limit the 
individual possibilities and provide an understanding of the form-function relations (ideas related to objects and signifiers and their uses) over time. Ontogenesis is the individual development over time.

Saxe used an interesting research procedure. By noting how the various genetic levels play out together, data collected at any one point in time could fit into one or other genetic mould. Furthermore, the interplay between social positional identities (e.g., teachers, people who had worked outside of the community, trade store owners, school children, adults without schooling) and the elaboration of mathematical goals were explored. Initially in the field he observed everyday activities, but he also asked people to specifically explain, for example, how they counted, which was visually demonstrated. They showed their system of tallying objects against parts of their body in a fixed order starting at the thumb (which is not a common starting point for body tally systems in PNG) of one hand to the small finger to the wrist, lower arm, inner elbow, upper arm, shoulder, ear, eye, nose and then across and down the other side, but going from the thumb to the small finger (not a mirror image, which is also unusual among the body-part tally systems [Lean, 1992; Owens, 2001]). Sometimes, people stopped at 20, the elbow of the other arm, because that matched with the 20 shillings or the pound (of the former colonial administration, Australia) and then adjusted to $\$ 2$ of the Australian decimal currency and finally to K2 of the PNG decimal currency (two kinaall amounts below this are coins). However, some people went back up the arm to the elbow representing 30 . Variation was evident in their systems and linguistic representations. Saxe then selected specific groups of people to support the view that certain experiences were likely to be associated with certain generations or experiences and to show that certain representations and ways of thinking were relatively common or valorised within a particular cohort or across cohorts (de Abreu, 2002).

Having come from an empirical tradition of psychology, Saxe carried out a range of studies, which he grouped into two areas. He gives details of each study. In general, assisting translators asked a set of fixed mathematical questions that linked into his developing genetic theory and the sociocultural circumstances of the participants. He had sufficient numbers of interviews to carry out both descriptive statistics (percentages mostly), illustrated graphically, and some chi-squared comparisons of results from different groups. (In his Brazilian study, he tended to compare across groups with descriptive statistics and analysis of variance tests.) The first study area related to the influence of the outside money economy found in trade-stores and used by others who had worked outside the community and to a lesser degree by those who sold garden produce and paid school fees only. The second study area related to the two-way influence of societal and school ways. On his visits, 20 years apart, he selected certain divergent groups to interview that illustrated different degrees of contact with outside influences. This also related to different visits across time, providing a check on the genetic explanation of change. However, he did not lose the serendipitous opportunities and the opportunity to review videotapes. Furthermore, he has shared his visual research on the internet (Saxe, n.d.).

I select here to summarise the findings from his first study on the money economy. The number of interviewees was large, nearly 80 in four categories, requiring walking considerable distances between trade-stores and villages. The addition and subtraction problems with or without 10 toea (10t) coins (still called shillings by some) were carefully counterbalanced. Each oral word problem was presented in terms of the trade-store buying and each number indicated on the body part equivalent. Again, we see Saxe's willingness to accept social practice in that people were coached by the translator to answer the question as there seemed to be little understanding of the question without this prompt for some people. The graphs present interesting differences in results, but a good proportion of all groups did well when coins were present, with more coaching of older people and schooled young adults for subtraction (quite a few had been systematic but gave the wrong addition answer). Saxe noted a common understanding of the story problem, without actually designating whether the problem was a difference or sum when coins were not present. Some people used words rather than body parts to match coins or amounts providing a more efficient response. For these people, it was clear that the body parts were a number system. This method was used by a significant number of people across groups except the older adults. The body-tallies led to an interesting compensation method where both arms were used with the shoulder (10) being a critical part in the designation and transfer of parts (e.g., 16 might be 10 on one arm and 6 on the other). This method was used by a few of the trade store owners and by very few others (mainly plantation returnees). In addition, by providing scores for advanced strategies that used body parts as a system to provide accuracy, Saxe was able to show a significant difference between groups. The differences, supported graphically, showed greater use by trade-store owners, with less use by plantation returnees and young adults, with no advanced strategies or use of the body-parts in a numerical system for older adults with problems without coins being present. The question remains whether this was lack of practice or indeed genetic development from social experiences.

Saxe indicated that hegemony and position of people such as trade store owners, teachers, missionaries, patrol officers and returning employees can have a noticeable impact on the development of practice. Thus Tok Pisin methods of referring to currency were universal, unlike the diversity of ways of naming and valuing currency. One particular word, $f u$, which might be understood as a complete group of plenty, was associated at different times and by different people with differing amounts in a consistent way. Thus the meaning and signification of $f u$ varied 
over time and cohorts, providing a strong example of his interlinked genetic theory. However, $f u$ is also associated with doubling. There is no explanation about how this developed, although it might link to the doubling of 10 to make 20 and 5 to make 10, both of which are marked by currency notes within the sociohistorical background of Oksapmin.

At the sociological level, in the case of the Oksapmin, there was the original isolation with links to neighbouring Telefol speakers (e.g., at Telefomin) who did use 27 body parts as a whole group, and talked about two whole groups and three whole groups. Then the Australian shillings and pounds arrived, followed by the Australian dollar and more but minimal contact with outsiders. Then the kina and toea became the currency, which was equivalent initially to the Australian dollar and cents, but soon gained and then lost value on the international market (a fact that concerned villagers only in terms of cash crops like coffee). However, more people were moving outside the Oksapmin region to earn money and mixing with more people. The Tok Pisin language, which uses a similar counting system to English, began to dominate in mathematics. With the body-part tally system, the tallies were not just names but ordered names. They were used for reasoning about size and they could be doubled. Hence the shoulder doubled matched the inner elbow on the other side, a practice coming with the K10 and K20 note exchanges. Saxe's theory does allow for the differences within a society and the changes that occur within a society. This was an area that Montiel and Macapagal (2011) were concerned about in looking at cultural identity. The degree to which there is continuity in change can be significant for the integrity of a person's identity of self-worth, by which they make effective decisions.
Saxe's background chapters on his personal journey and his visits to Oksapmin and the historical background of currency changes in Papua New Guinea together with the carefully developed reports of each study and the overall linkage with his theoretical perspective make this an important contribution to psychology especially within the Pacific Rim region, and a crucial reference for those in Papua New Guinea interested in mathematics, education, cognition, psychology, anthropology and/or sociology.

\section{References}

de Abreu, G. (2002). Towards a cultural psychology perspective on transitions between contexts of mathematical practices. In G. de Abreu, A. Bishop , \& N. Presmeg (Eds.), Transitions between contexts of mathematical practices (pp. 170-189). Dordrecht, The Netherlands: Kluwer.

Lean, G. (1992). Counting systems of Papua New Guinea and Oceania. (Unpublished $\mathrm{PhD}$ dissertation). University of Technology., Lae, Papua New Guinea. Retrieved from http://www.uog.ac.pg/glec/

Montiel, C., \& Managal, M.A. (2011). Commentary: Nuancing the meaning of cultural diversity. Journal of Pacific Rim Psychology, 5(2), 81-84.

Owens, K. (2001). The work of Glendon Lean on the counting systems of Papua New Guinea and Oceania. Mathematics Education Research Journal, 13(1), 47-71.

Saxe, G. (n.d.). Cultural development of mathematical ideas. Retrieved from http://www.culturecognition.com/

Saxe, G. (1991). Culture and cognitive development: Studies in mathematical understanding. Hillsdale, NJ: Lawrence Erlbaum. 